\title{
Boosting the performance of distributed optical fiber sensors based on adaptive decoder
}

\author{
Xizi Sun ${ }^{1}$, Zhisheng Yang ${ }^{2, *}$, Xiaobin Hong, ${ }^{1, *}$, Simon Zaslawski ${ }^{2}$, Sheng Wang ${ }^{1}$, Jian Wu ${ }^{1}$ and Luc \\ Thévenaz ${ }^{2}$ \\ ${ }^{1}$ State Key Laboratory of Information Photonics \& Optical Communications, Beijing University of Posts and Telecommunications, Beijing \\ 100876, China \\ ${ }^{2}$ EPFL Swiss Federal Institute of Technology, Institute of Electrical Engineering, SCI STI LT, Station 11, CH-1015 Lausanne, Switzerland \\ Corresponding author: zhisheng.yang@epfl.ch; xbhong@bupt.edu.cn
}

\begin{abstract}
An adaptive decoder is proposed to derive the fiber single-pulse response. This experimentally simple technique simultaneously improves the measurement time and the noise level, boosting to an uncommon degree the overall performance of distributed fiber sensors. (C) 2018 The Author(s) OCIS codes: (060.2370) Fiber optics sensors; (290.5900) Scattering, stimulated Brillouin.
\end{abstract}

\section{Introduction}

Distributed optical fiber sensors can offer promising performance for monitoring several physical quantities such as temperature and strain along an optical fiber. Making use of Rayleigh, Brillouin or Raman scattering, distributed fiber sensing can be implemented in various domains (e.g., time, frequency or correlation domain) [1]. Among them the classical time-domain techniques based on the concept of optical time-domain reflectometry (OTDR) have been widely studied over the last decades, in which the information along the fiber is spatially resolved by analyzing the backscattered signal from an optical pulse [1]. It has been demonstrated that the most critical parameter scaling the performance of such sensors is the signal-to-noise ratio (SNR) [2]. For a given spatial resolution, the SNR is proportional to the input pump power, which is ultimately limited by nonlinear effects in the sensing fiber. To further enhance the SNR, solutions based on optical pulse coding, such as Golay [3], Simplex [4] and cyclic codes [5], are extensively developed. Whilst the power of each pulse is strictly kept below Kerr threshold, a coding gain (i.e., SNR improvement) being half of the square-root of the code length can be achieved [3-5]. Conceptually, optical pulse coding techniques can keep the same experimental layout, featuring similar measurement times when compared to a single-pulse approach. It is therefore considered as one of the most efficient way to boost the performance of distributed sensors. However, additional devices have to be practically inserted to compensate the transient effects in erbium-doped fiber amplifier (EDFA) [6]. Both Golay and Simplex codes require to launch and store hundreds of coded sequences and traces, so that both the measurement and postprocessing turn time-consuming, even though the total number of averaged raw acquisitions is unchanged. Cyclic codes require to only launch the coding sequence once, though the code length is limited by the fiber length [5].

In this paper, inspired by the constrained least-square (CLS) filtering first employed in the field of image restoration [7], an adaptive decoder is proposed for distributed sensing to decode the data encoded by only one sequence. This decoder enables a measurement and post-processing time similar to a single-pulse based distributed sensor. Moreover, the parameters of such a decoder can be adaptively optimized for a given envelop of the code sequence, thus turning immune to the pulse power non-uniformity imposed by EDFA transient effects, with no need to insert additional devices. Finally, by properly designing the code sequence, the SNR improvement can be higher than that of traditional coding techniques, depending on the ratio between signal and detector bandwidths. To the best of our knowledge, the proposed technique is the fastest in speed, the simplest in implementation and the highest in SNR improvement, only compromised by distortions that can be made indiscernible.

\section{Principle}

Let define $s_{k}$ and $h_{k}$ the sampled time-domain single pulse profile and fiber impulse response, respectively. In a linear system (e.g., BOTDA, BOTDR, ROTDR and non-coherent OTDR). The ideal time-domain single-pulse response (backscattered trace) $r_{k}^{s}$ along with its spectrum $R_{k}^{s}$ can be typically described as:

$$
r_{k}^{s}=s_{k} \otimes h_{k} \stackrel{F F T}{\longrightarrow} R_{k}^{s}=S_{k} H_{k}
$$

where $\otimes$ denotes the convolution product, $S_{k}$ and $H_{k}$ corresponding to the spectra of $s_{k}$ and $h_{k}$ obtained via a Fourier transform, respectively. In case of a square-shaped input pulse, the corresponding $S_{k}$ exhibits a sinc profile with a bandwidth determined by the spatial resolution, as exemplified by the red curve shown in Fig. 1(a), in the case of a $100 \mathrm{~ns}$ pulse and $5 \mathrm{~ns}$ sampling interval. When a coded pulse sequence $\mathrm{c}_{k}$ is used and considering the contribution of noise ( $e_{k}$ in time domain and $E_{k}$ in frequency domain), the backscattered signal $r_{k}^{c}$ representing the coded response is expressed as:

$$
r_{k}^{c}=\mathrm{c}_{k} \otimes h_{k}+e_{k}=\left(s_{k} \otimes \delta_{k}\right) \otimes h_{k}+e_{k} \stackrel{F F T}{\longrightarrow} R_{k}^{c}=S_{k} \Delta_{k} H_{k}+E_{k}=C_{k} H_{k}+E_{k}
$$


where $\delta_{k}$ is the coding sequence expressed as a series of delta function, $\Delta_{k}$ is the frequency domain representation of $\delta_{k}$, and $\mathrm{c}_{k}=\left(s_{k} \otimes \delta_{k}\right)$. In order to retrieve the single-pulse fiber response $r_{k}^{s}$ in Eq. (1), the decoding technique for Eq. (2) may consist in taking a decoder $1 / \Delta_{k}$ represented in the frequency domain as:

$$
r_{k}^{d s}=\operatorname{IFFT}\left(R_{k}^{d s}\right)=\operatorname{IFFT}\left(\frac{R_{k}^{c}}{\Delta_{k}}\right)=\operatorname{IFFT}\left(S_{k} H_{k}+\frac{E_{k}}{\Delta_{k}}\right)=r_{k}^{s}+\operatorname{IFFT}\left(\frac{E_{k}}{\Delta_{k}}\right)
$$

where $r_{k}^{d s}$ represents the decoded single-pulse response of the system. Note that although the signal term $r_{k}^{s}$ can be perfectly recovered by Eq. (3), the original noise $E_{k}$ may be significantly magnified by the term $1 / \Delta_{k}$ when $\Delta_{k}$ is far less than 1, depending on the specific distribution of spectral components determined by the particular code sequence. Intuitively, $\Delta_{k}$ must contain the smallest number of zero amplitude spectral components, suggesting a non-periodic time-domain code that typically corresponds to a standard pseudo-random binary sequence (PRBS). Fig. 1(b) compares two $1 / \Delta_{k}$ distributions in the cases of a 127-bit PRBS code (red) and a periodical ' 1,0 ' code with the same bit length (blue), the former exhibits much less spectral spikes that magnify the noise. Nevertheless, it can be observed that even in PRBS case, the amplitudes of some spectral components are still larger than 1, degrading the SNR performance. Aiming at solving this problem while keeping the advantage of retrieving the single-pulse response via only one coded trace, and inspired by the concept of CLS filter, a novel decoder based on the functional expression $\Delta_{k}^{*} /\left(\left|\Delta_{k}\right|^{2}+\alpha P_{k}\right)$ is here proposed, where $\alpha$ and $P_{k}$ are defined as the filtering coefficient and the filtering function, respectively. Using this proposed decoder, the retrieved single-pulse fiber response in frequency domain is derived as:

$$
R_{k}^{d s}=\frac{\Delta_{k}^{*} R_{k}^{c}}{\left|\Delta_{k}\right|^{2}+\alpha\left|P_{k}\right|}=\frac{\Delta_{k}^{*} S_{k} \Delta_{k} H_{k}+\Delta_{k}^{*} E_{k}}{\left|\Delta_{k}\right|^{2}+\alpha\left|P_{k}\right|}=\frac{R_{k}^{s}}{1+\alpha\left|P_{k}\right| /\left|\Delta_{k}\right|^{2}}+\frac{E_{k}}{\Delta_{k}\left(1+\alpha\left|P_{k}\right| /\left|\Delta_{k}\right|^{2}\right)}
$$

where $\left|P_{k}\right|$ is a high-pass filter, as shown in Fig. 1(a), which minimizes the term $\alpha\left|P_{k}\right| /\left|\Delta_{k}\right|^{2}$ within signal bandwidth $B_{s}$ (bandwidth of $S_{k}$ ) while maximizing the integration of the term $\Delta_{k}\left(1+\alpha\left|P_{k}\right| /\left|\Delta_{k}\right|^{2}\right.$ ) over the detection bandwidth $B_{d}$. By carefully adjusting the value of $\alpha$, the single-pulse response can be recovered with a minimum distortion at maximum SNR.
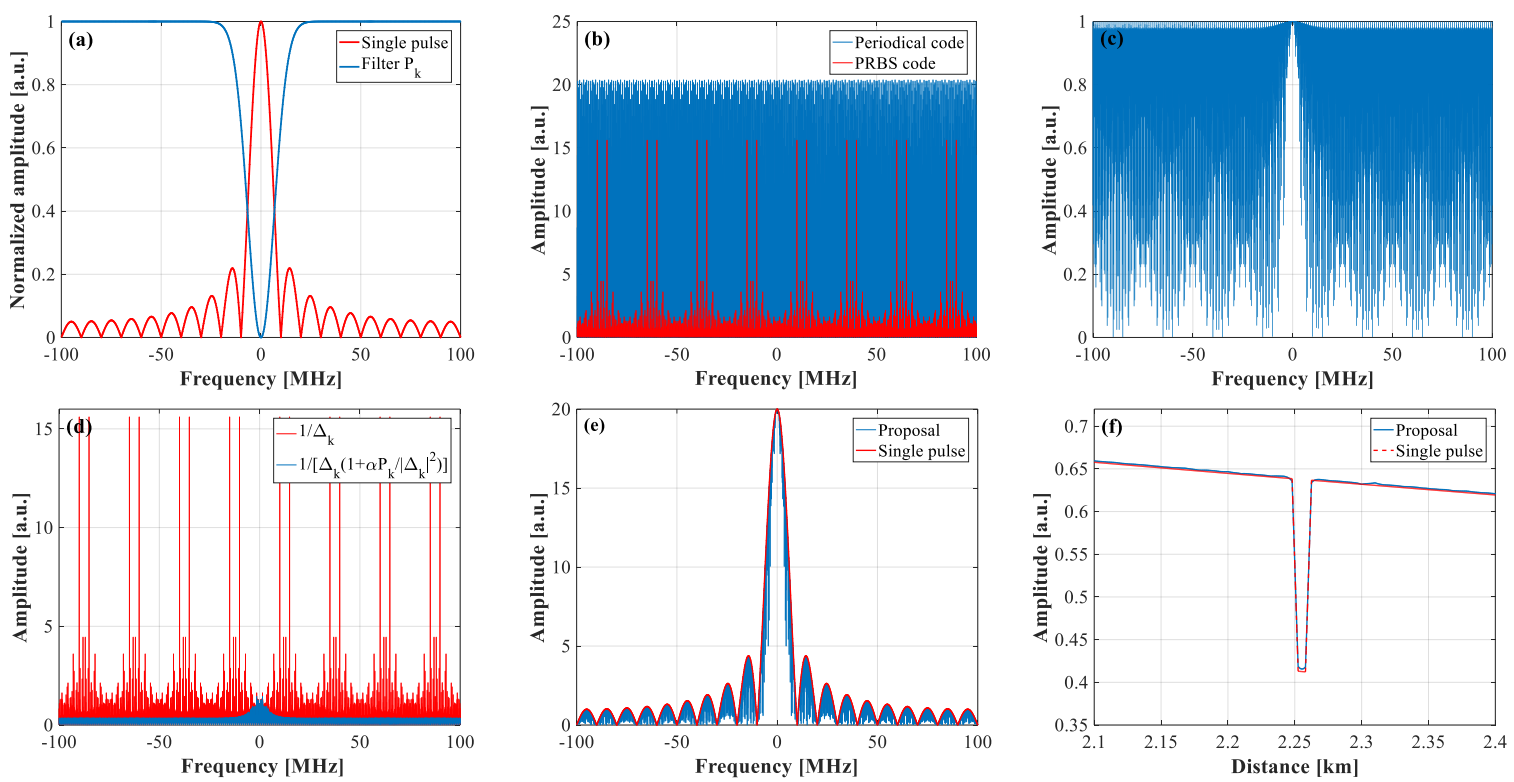

Fig. 1 Simulations based on $20 \mathrm{~ns}$ pulse and $200 \mathrm{MS} / \mathrm{s}$ sampling rate. (a) the spectrum of sampled single pulse and high-pass filter; (b) the $1 / \Delta_{k}$ distributions of a 127-bit PRBS code and a periodical ' 1,0 ' code. (c) the decoded transfer function for signal; (d) the transfer function of noise with and without adaptive decoder; (e) the decoded interrogating function; (f) the decoded single-pulse response.

Under the optimized condition, the term $1 /\left(1+\alpha\left|P_{k}\right| /\left|\Delta_{k}\right|^{2}\right)$ in Eq. (4), which acts as signal transfer function, is close to 1 at most spectral positions, as illustrated in Fig. 1(c), meaning that most spectral components of the signal pass through. Meanwhile, the term $1 /\left[\Delta_{k}\left(1+\alpha\left|P_{k}\right| /\left|\Delta_{k}\right|^{2}\right)\right]$ that accounts for noise rejection, is greatly reduced with respect to the case described by Eq. (3), as shown in Fig. 1(d). This way, the spectrum of the 
equivalent interrogation function $\left|\Delta_{k}\right|^{2} S_{k} /\left(\left|\Delta_{k}\right|^{2}+\alpha\left|P_{k}\right|\right)$ is in good agreement with the spectral profile of a single pulse, as depicted in Fig. 1(e), demonstrating that the proposed technique can keep the essential information from the signal. For further demonstration, performance in the time domain is investigated by simulating the response around an artificially created hotspot. As shown in Fig. 1(f), the decoding response using the optimized decoder (blue) matches the ideal single-pulse response (red) with minor distortion. Moreover, it can be expected that most of the noise outside $B_{s}$ is removed (i.e., the noise is reduced by $\sqrt{B_{s} / B_{d}}$ ), while the original coding gain $\sqrt{L} / 2$ is kept, offering an overall SNR improvement of $\sqrt{L \times B_{s} / B_{d}} / 2$, outperforming classical coding techniques.

\section{Experimental results and discussion}

For the sake of simplicity, an experimental configuration strictly based on the classical non-coherent OTDR is setup to demonstrate the feasibility of the proposed approach. Firstly, experiments are carried out over a 4-km long sensing fiber with an abrupt attenuation of $2.5 \mathrm{~dB}$ at a median position. To validate the feature that the code length can be longer than the fiber length, we deliberately employed a relatively wide pulse width of $100 \mathrm{~ns}$, corresponding to a $10 \mathrm{~m}$ spatial resolution. A unipolar PRBS code sequence is encoded using a return-to-zero (RZ) format, anticipating its potential use in BOTDA. The code length is 127 bits for a RZ duty cycle of $10 \%$. The coded pulse sequence covers a range of $12.7 \mathrm{~km}$, i.e. much larger than the fiber length. The backscattered signals are acquired by using 1024 averages. At the receiver, the signal is acquired by a $75 \mathrm{MHz}$ bandwidth photodiode at a $200 \mathrm{MS} / \mathrm{s}$ sampling rate.

To study the trade-off between the ultimate SNR improvement and the spatial resolution degradation, measurements are first realized without EDFA, thus ensuring that the profile of the coded sequence is flat, i.e. each code bit counts for an equal contribution. The decoding performed by a Matlab 2015b routine, using an i76500U CPU, took only $10 \mathrm{~ms}$, indicating its potential use for on-line processing dynamic measurements. Fig. 2(a) shows the decoded curve (red) when using an optimized $\alpha=20$, along with the single-pulse responses at 1024 (blue) and 32768 averages (black). The SNR in the latter case is equivalent to the one that could be ideally obtained using 127-bit unipolar Simplex coding or 128-bit Golay coding, indicating that the present technique outperforms other methods. Fig. 2(b) compares the SNR in the aforementioned 3 conditions quantitatively, where the red curve shows $12 \mathrm{~dB}$ (15.8 times in linear scale) SNR improvement over the single-pulse response with the same average, and 4.5 dB SNR improvement over traditional coding gain. This is perfectly consistent with the theory presented in Section $2(\sqrt{127 \times 75 / 10} / 2=15.4)$. Actually, by further increasing the value of $\alpha$, the SNR improvement can be even larger (see Fig. 2(c)) since it further narrows the filtering bandwidth. However, this gives rise to signal over-smoothing thus compromising the spatial resolution, as can be clearly seen in Fig. 2(d).
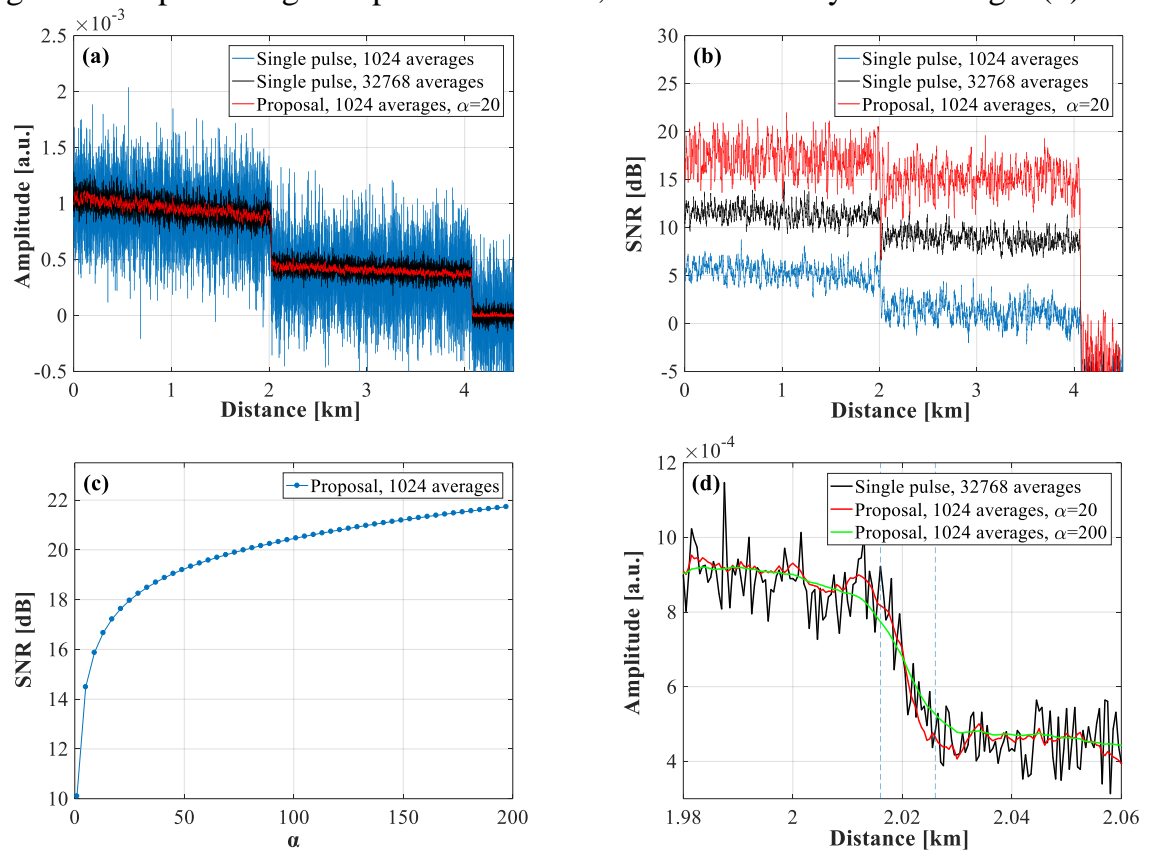

Fig. 2 (a) The measured and decoded traces along the sensing fiber; (b) SNR of each trace; (c) SNR of the decoded trace versus $\alpha$; (d) The trace over a fiber section from $1.98 \mathrm{~km}$ to $2.06 \mathrm{~km}$.

Then an EDFA is inserted into the system, aiming at investigating the adaptability of the technique to nonuniformity of the pulse power in the sequence. The optical power of the first coding pulse is adjusted to match the 
pulses power without EDFA, to compare the difference in terms of SNR performance. The coding sequence resulting from the EDFA transient effect is plotted in Fig. 3 (a). Defining this EDFA-amplified sequence as $c_{k}$ to carry out the decoding based on Eq. (4), the decoded trace, shown as the red curves in Fig. 3(b) and (c), remains non-distorted. This validates that the proposal method can be applied in the case of an uneven pulse power distribution along the sequence, being crucially advantageous over Simplex, Golay and Cyclic codes.
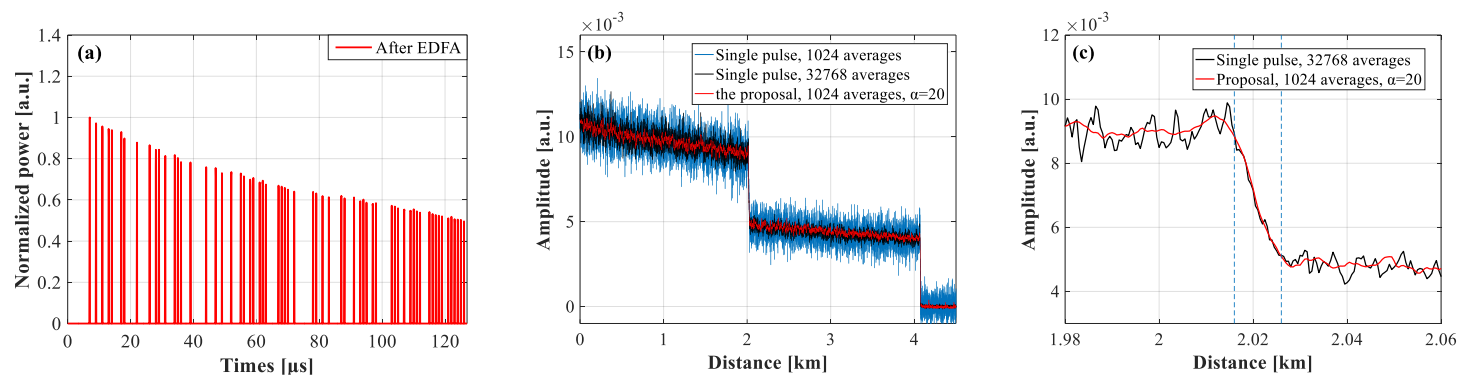

Fig.3 (a) Normalized 127-bit PRBS coded optical pulse sequence at the EDFA output. (b) The measured and decoded traces along the sensing fiber when the EDFA is used. (c) The trace over a fiber section from $1.98 \mathrm{~km}$ to $2.06 \mathrm{~km}$ when EDFA is used.

Finally, a $50 \mathrm{~km}$ sensing fiber is tested using a $20 \mathrm{~ns}$ pump pulse, and a 511-bit RZ PRBS code with 10\% duty cycle, respectively, under a sample rate of $500 \mathrm{MS} / \mathrm{s}$. The averaging numbers are 2048 for the coded trace, 2048 and $262144(=2048 \times 512 / 4)$ for the single pulse. The retrieved time-domain traces in each case are shown in both linear (Fig. 4(a)) and logarithmic (Fig. 4(b)) scales, respectively. Theoretically, the proposed method (red) can provide $\sqrt{75 / 50}=1.1$ times better performance than the single-pulse trace with 262144 averages (black). However, it is widely compromised by the lowest total energy in the pulse sequence power caused by the EDFA transient effect. The results demonstrate the technique can at least keep the traditional coding gain, with much faster measurement and post-processing time, as well as a simpler interrogating configuration.
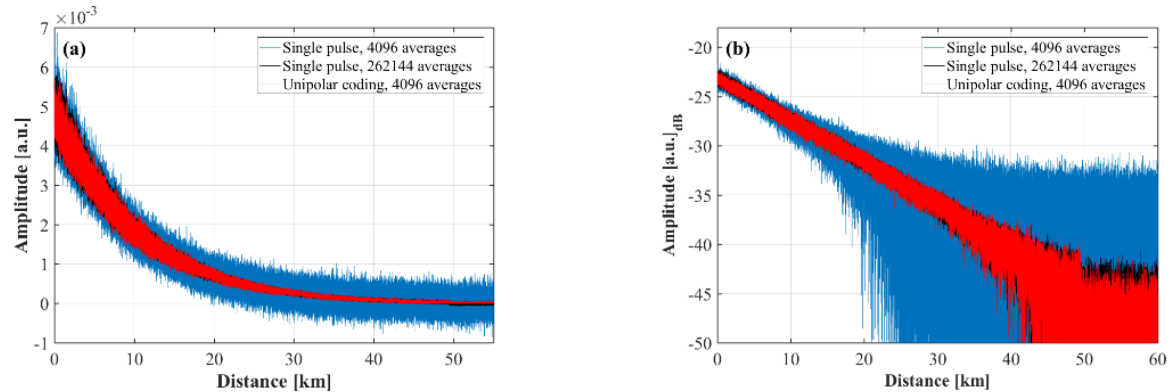

Fig.4. The measured and decoded traces along the sensing fiber for single-pulse and unipolar coding method in (a) linear scale; (b) logarithmic scale.

\section{Conclusion}

In this work a one-shot coding technique with a dedicatedly designed adaptive decoder has been proposed and experimentally investigated using classical non-coherent OTDR. It has been demonstrated that the proposed method can offer several advantages: 1) adaptability to non-uniform power along the pulse sequence, such as that imposed by an EDFA, simplifying the experimental layout; 2) higher SNR improvement compared to the traditional coding technique; 3) launching of a one-shot coded sequence and fast decoding, with potential application for dynamic sensing. All the aforementioned advantages come at the expense of decoding distortion, which can be turned indiscernible if maintained below the noise level by a proper parameters adjustment.

Acknowledgement: National Key R\&D Program of China under Grant (2017YFB0405500); Swiss Commission for Technology and Innovation (18337.2 PFNM-NM).

\section{Reference}

[1] B. Culshaw, and A. Kersey, "Fiber-Optic Sensing: A Historical Perspective," J. Lightw. Technol. 26, 1064-1078 (2008).

[2] M. A. Soto and L. Thévenaz, "Modeling and evaluating the performance of Brillouin distributed optical fiber sensors," Opt. Express 21, 31347-31366 (2013).

[3] M. Nazarathy, et al., "Real-time long range complementary correlation optical time domain reflectometer," J. Lightw. Technol. 7, 24-38 (1989).

[4] M. D. Jones, "Using simplex codes to improve OTDR sensitivity," IEEE Photonic Technol. 5, 822-824 (1993).

[5] M. A. Soto, et al., "Raman-based distributed temperature sensor with 1m spatial resolution over 26km SMF using low-repetition-rate cyclic pulse coding," Opt. Lett. 36, 2557-2559 (2011).

[6] M. A. Soto, et al., "Long-range Brillouin optical time-domain analysis sensor employing pulse coding techniques," Meas. Sci. Technol. 21, 094024 (2010).

[7] R. C. Gonzalez, R.E. Woods., Digital Image Processing, New Jersey, Prentice Hall, 266-269 (2002). 